\title{
Trans-Photospheric Wave Leakage in Be Stars
}

\author{
Rich Townsend \\ Department of Physics \& Astronomy, University College London, \\ Gower Street, London WC1E 6BT, UK
}

\begin{abstract}
The phenomenon of trans-photospheric wave leakage in stars undergoing non-radial pulsation is investigated, with an emphasis on its significance for Be stars. It is demonstrated that a symmetry breaking in line-profile variability may be used as a diagnostic for such wave leakage in these stars.
\end{abstract}

\section{Introduction}

Recent observations of line-profile variations (lpv) in early-type stars, and in particular Be stars, appear to suggest that a significant proportion of these systems undergo non-radial pulsation (NRP). It is therefore natural to enquire whether such NRP plays a significant role in the episodic disk formation associated with the Be phenomenon, and, if so, what physical mechanism underpins the putative NRP-Be connection.

One such mechanism, which can promote disk formation by transporting angular momentum from the star out into the circumstellar environment, is the phenomenon of trans-photospheric wave leakage. The possibility of wave leakage has previously been overlooked in pulsation studies, since the commonly-adopted outer mechanical boundary condition formulated by Dziembowski (1971) contains the assumption that the Lagrangian pressure perturbation $\delta p$ is radially constant at the stellar surface (ie, $\mathrm{d} \delta p / \mathrm{d} r \rightarrow 0$ ). This assumption implies that pulsation has the character of standing waves at the surface, and therefore no wave leakage is possible. However, Ando \& Osaki (1975) presented an alternative formulation for the boundary condition which relaxes this assumption and allows, under certain conditions, for the leakage of pulsation waves through the photosphere. This formulation is described briefly in the following section, and its significance to Be stars is investigated in section 3 , whilst section 4 examines the consequences of wave leakage for lpv, and section 5 summarizes the findings.

\section{Wave Leakage}

Ando \& Osaki (1975), in their formulation of the surface boundary condition, assumed that all of the coefficients in the linear adiabatic pulsation equations are constant near the stellar surface. This assumption permits solutions to the equations of the form $\xi_{r}, p^{\prime} \sim r^{\lambda}$, where $\xi_{r}$ and $p^{\prime}$ are the radial Lagrangian displacement and Eulerian pressure perturbation respectively. The propagation exponent $\lambda$ is a constant which governs the nature of the pulsation at the stellar 
surface; its value depends on two cut-off frequencies $\omega_{l}$ and $\omega_{\mathrm{h}}$ which are determined from Ando \& Osaki's formulation. If the pulsation frequency $\omega$ falls between these two cut-off frequencies, such that $\omega_{1}<\omega<\omega_{\mathrm{h}}$, then $\lambda$ is real and negative, and the amplitude of $\xi_{r}$ and $p^{\prime}$ decays exponentially with radius, with the corresponding phase remaining spatially constant. This situation corresponds to evanescent pulsation, where waves are completely reflected at the stellar surface in a manner similar to that found using Dziembowski's formulation.

However, if either $\omega<\omega_{1}$ or $\omega>\omega_{\mathrm{h}}, \lambda$ is complex, and the phase of $\xi_{r}$ and $p^{\prime}$ becomes a function of radius. Such a spatial phase dependence is a fundamental characteristic of propagating waves; therefore, in such cases, the stellar surface is unable to reflect completely pulsation waves originating from the interior, and to a certain degree wave energy will leak through the photosphere and out into the circumstellar environment in the form of travelling waves.

\section{Application to Be Stars}

The characteristically long variability periods observed in Be stars undergoing NRP indicates that they oscillate predominantly in low-frequency gravity (g) modes. Therefore, the appropriate criterion for wave leakage to occur in such systems is that the pulsation frequency $\omega$ is smaller than the lower cut-off frequency $\omega_{1}$. This cut-off frequency depends on, amongst other quantities, the pressure scale height at the stellar surface; longer pressure scale heights (corresponding to more extended surface regions) generally lead to larger values of $\omega_{1}$. If this result is combined with the requirement of hydrostatic equilibrium (whereby the pressure scale height is inversely proportional to the gravity), it can be concluded that stars with a low surface gravity are the ones most likely to exhibit wave leakage.

This characteristic of wave leakage is particularly pertinent to Be stars, since these systems are known to rotate rapidly, typically at $50 \%$ of the critical velocity, and therefore have low (effective) surface gravities around equatorial regions due to strong centrifugal forces. It is therefore a strong possibility in many pulsating Be stars that rotation will raise $\omega_{l}$ sufficiently for one or more pulsation modes to leak through the photosphere preferentially around the stellar equator.

As Unno et al. (1989) have demonstrated, pulsation waves can transport not only energy through the stellar surface, but also angular momentum. The direction of this angular momentum flux depends on the azimuthal direction of propagation of pulsation modes in the co-rotating frame of reference - prograde and retrograde modes lead to outward and inward angular momentum fluxes, respectively. It is not yet clear what proportion of NRP modes in Be stars are prograde or retrograde, so it cannot be said for sure whether wave leakage would promote or hinder the formation of a Keplerian disk by angular momentum transport to/from circumstellar material.

However, such a debate is somewhat incidental to the central question of whether wave leakage does actually occur in Be stars. The only means of resolving this issue is through a confrontation between theory and observation; 


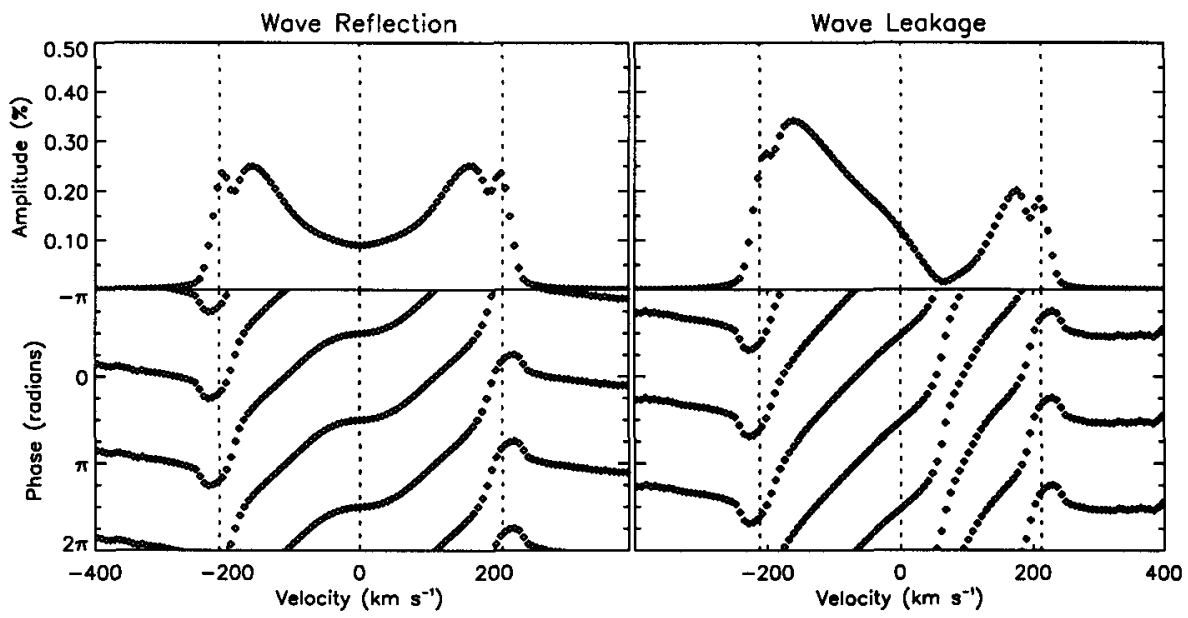

Figure 1. The amplitude (as a percentage of the continuum) and phase of lpv across the He I $6678.14 \AA$ line profile, calculated with wave reflection (left-hand panel) and wave leakage (right-hand panel) at the stellar surface.

accordingly, the following section investigates what effect wave leakage will have on lpv in a typical Be star undergoing NRP.

\section{Effects on Line-Profile Variability}

In situations where wave leakage occurs, both $\xi_{r}$ and $p^{\prime}$ will be complex, due to the complex nature of the propagation exponent $\lambda$ mentioned in section 2 . In addition, as result of Ando \& Osaki's boundary-condition formulation, there will be a phase difference between these two quantities at the stellar surface, which depends on the degree of leakage occurring; in cases where the leakage is significant, this difference can approach $\pi / 2$ radians. Such a condition does not occur in the case of complete wave reflection, where both $\xi_{r}$ and $p^{\prime}$ are always in phase.

Since the horizontal Lagrangian displacement $\xi_{h}$ is proportional to $p^{\prime}$ (e.g., Unno et al. 1989), the effect of the phase difference is to shift the pattern of horizontal displacements and velocity fields around the stellar surface, relative to the radial displacements and velocity fields, by as much as a quarter-rotation. Furthermore, the dependence of temperature perturbations on $p^{\prime}$ (e.g., Buta \& Smith 1979) will mean that they too will be shifted around the surface by a related amount.

A combination of these pattern-shifting effects will lead to a change in the morphology of the lpv exhibited by a pulsating star when wave leakage occurs. To demonstrate this phenomenon, two 24-spectra time-series covering one (observer's-frame) period of an $l=4$ prograde sectoral pulsation mode were calculated for the He I $6678.14 \AA$ line profile using the lpv modelling code BRUCE (Townsend 1997). The stellar parameters were chosen to correspond 
loosely to those of the prototype Be star $\gamma$ Cas, and a observer's period of 2.8 hours was adopted, corresponding to the g-mode pulsation mentioned in the preceding section. Whilst the first time-series was calculated in the usual way (ie, under the assumption of complete wave reflection at the surface), the second was calculated with the inclusion of a $\pi / 2$-radian phase difference between $\xi_{r}$ and $p^{\prime}$, as described above, to simulate the effects of significant wave leakage.

Fig. 1 shows the amplitude and phase of the lpv corresponding to the two time-series calculated. It is immediately apparent that the effect of wave leakage is both to distort the phase of the variations, and, more importantly, to break the velocity symmetry (with respect to the line-center) of the variability amplitude distribution. This symmetry is inherent in the lpv of any intrinsically-symmetric line profile calculated under the assumptions of adiabaticity and complete wave reflection, as is evidenced by the left-hand panel.

The only other mechanism by which this symmetry breaking may occur is through non-adiabatic effects; however, these effects are only significant in the case of extremely non-adiabatic pulsation such as secular instability, which is not expected in the majority of stars (Unno et al. 1989). Therefore, it is proposed that the symmetry breaking of the type shown in the right-hand panel of Fig. 1 may be used as an observational diagnostic for wave leakage in Be stars.

\section{Summary}

The principal idea behind the work presented herein is to indicate what the observational signature of trans-photospheric wave leakage will be, if leakage does indeed occur in Be stars. Whilst no attempt has been made to investigate in detail how such leakage might play a part in the Be phenomenon, it appears at first glance to be a promising candidate mechanism for transporting angular momentum out to a circumstellar disk. However, even though further theoretical investigation is required, it should be possible to undertake an observational search for wave leakage now, using the discussed symmetry breaking as a diagnostic. The author hopes that such a search will be undertaken in the near future.

\section{References}

Ando, H., \& Osaki, Y. 1975, PASJ 27, 581

Buta, R.J., \& Smith, M.A. 1979, ApJ 232, 213

Dziembowski, W. 1971, Act. Ast. 21, 289

Townsend, R.H.D. 1997, MNRAS 284, 839

Unno, W., Osaki, Y., Ando, H., Saio, H., \& Shibahashi, H. 1989, Nonradial Oscillations of Stars (Tokyo: University of Tokyo Press) 